\title{
ESTATÍSTICAS PARA DETECÇÃO DE BOTS EM REDES SOCIAIS
}

\author{
Caio Henrique Assad Racy, Kelton Augusto Pontara da Costa, Miguel José das Neves \\ e Thiago José Lucas \\ Unesp - Universidade Estadual Paulista "Júlio de Mesquita Filho", Bauru, São Paulo, Brasil
}

\begin{abstract}
RESUMO
A presente pesquisa é um estudo sobre as estatísticas para detecção de social bots, com estudos voltados para a análise de bots de seguidores falsos conhecidos. Esta pesquisa tem como objetivo analisar por meio de métodos estatísticos de média, desvio padrão e variância, além do uso do Método de Regressão Linear Simples na possibilidade e usabilidade de detectar bots maliciosos mencionados. De acordo com o estudo bibliográfico desenvolvido, é possível mostrar como a estatística pode ser útil nas diferenças entre usuários reais de bots, além de poder melhorar métodos e usar vários outros para refinar a pesquisa. Os métodos utilizados na pesquisa foram principalmente quantitativos, a partir da análise das características de um banco de dados pronto. Por fim, a pesquisa constatou que a estatística é muito útil para analisar esse tipo de bot, com resultados extremamente diferentes entre os usuários.
\end{abstract}

\section{PALAVRAS-CHAVE}

Estatísticas, Redes Sociais, Detecção, Social Bots

\section{INTRODUÇÃO}

Ataques cibernéticos ocorrem constantemente e tem o intuito de capturar informações e utilizar-se de outros sistemas para ganho pessoal. Nesse contexto surge o conceito de bot, palavra inglesa derivada de "robot" (robô) que significa software de automatização de procedimentos e que no momento atual, seu foco principal é na aplicação maliciosa para obtenção de dados de usuários e empresas.

De acordo com Qamar, Karim e Chang (2019), um programa de computador é considerado malicioso quando se dispõe de mecanismos de comunicação com o invasor, permitindo o controle remotamente do sistema, sendo capaz de se propagar automaticamente e com isso explorar as mais variadas vulnerabilidades existentes.

Os bots são mais difíceis de serem detectados, em relação aos outros malwares, estes podem ser controlados remotamente, ficando em estado de hibernação até o momento oportuno, quando o invasor necessita realizar algum comando ou mesmo que de modo automático, procedendo durante o modo de espera do computador. É o que afirmam Carna (2012) e Distils (2018). Os autores ainda complementam afirmando que as principais ações maliciosas causadas pelos bots retratam ataques de negação de serviço (DDoS), propagação de códigos maliciosos, coleta de informações, envios de spam e camuflagem da identidade do invasor. Empresas preocupadas com o crescimento de bots maliciosos, tem investido em pesquisas no intuito de aprimorar as ferramentas de detecção.

Alguns trabalhos relevantes são descritos a seguir: em Broniatowski et al. (2018) pode-se observar um importante cenário onde bots atuaram na Rússia com o objetivo de divulgar mensagens antivacina (saúde pública) impactando diretamente o debate público acerca do tema, o que documenta a amplitude dos problemas que podem ocorrer em virtude da existência de bots em redes sociais. Já em Campos et al. (2018) um experimento onde os autores obtiveram um excelente resultado (mais de $94 \%$ de acurácia) utilizando padrões da linguagem formal como objeto de classificação de contas no Twitter. Os testes foram realizados utilizando o algoritmo Randon Forest em duas bases de dados; de acordo com os autores é capaz de detectar bots com uma precisão de $94 \%$ de acerto a sincronicidade. Outro trabalho relevante pode ser observado em Chavoshi et al. (2016a) onde os autores documentam um estudo realizado observando o fato de que seres humanos não podem ser altamente síncronos em relação às suas atividades nas redes sociais. O trabalho 
referenciado por Chavoshi et al. (2016b), de forma diferente da maior parte dos outros esforços, foca em detectar bots não tratando as contas individualmente, mas observando a correlação entre diferentes usuários. O método obteve uma precisão de $94 \%$ para sucesso na detecção de bots.

Um trabalho que foca em detecção de bots em tempo real apresentado por Chen et al. (2017) foi desenvolvido por meio da criação de um protocolo, numa arquitetura que independe de linguagem, tópicos e palavras-chave, de forma não supervisionada. A ferramenta BotOrNot desenvolvida por Davis et al. (2016) é descrita como um sistema de avaliação online que pode classificar uma determinada conta como pertencente a um legítimo ser-humano ou não utilizando para tal mais de mil recursos. Um trabalho proposto por Efthimion et al. (2018) aborda um algoritmo de aprendizado de máquina que analisa comprimento do nome de usuários, taxa de repostagem, padrões temporais, expressão de sentimentos, relação seguidores e amigos além da variabilidade das mensagens para detecção de bots. O método é bastante eficaz pois apresentou nos testes uma taxa de erro de apenas 2,25\%. O trabalho descrito por Haidermota e Pansare (2018) propõe um modelo de classificação de usuários utilizando aprendizado de máquina com o objetivo de analisar diferentes características entre humanos e bots. Os autores focaram nos méritos das postagens no Twitter e propriedades das contas. Outro importante estudo Howard et al. (2016) realizado durante o primeiro debate presidencial nas eleições norte-americanas de 2016. O esforço detectou diferentes tipos de comportamentos em relação ao candidato apoiado. Os resultados apontam para o favorecimento de um determinado candidato em relação ao outro por meio de atividade de bots, o que, assim como o proposto por Broniatowski et al. (2018), demonstra como os bots podem impactar a opinião pública. Morstatter et al. (2016) propuseram um método de detecção de usuários e bots que incrementa a curva precision/recall quando comparado a outros métodos, no que tange aos resultados. O mérito principal do trabalho está na quantidade de bots que são detectados em comparação a outras abordagens que refletiam o estado da arte quando da realização dos experimentos.

Este trabalho, tem como foco abordar métodos estatísticos para conseguir detectar representativamente bots maliciosos que trafegam pela rede. Como existem vários tipos de bots, optou-se por utilizar a análise em bots difundidos nas redes sociais, conhecidos por "Social Bots", que podem ser considerados maliciosos quando tentam se passar por humanos a fim de influenciar opiniões e padrões nas redes sociais e disseminar notícias falsas.

Para o estudo utilizou-se uma base de dados com diversas contas da plataforma Twitter, a fim de analisar os aspectos humanos e verificar se é possível fazer uma análise estatística sobre o tipo de bot, com vertente em outros subtipos de bots:

Fake Followers: utilizados para espalhar falsa popularidade na rede, sendo comprados por pessoas que desejam aumentar seus números de seguidores, não possuem muita interatividade, mas servem para testar os métodos estatísticos, e utilizados nesta pesquisa como centro no estudo em questão.

Spam Bots: intuito é passar e divulgar informações nas redes sociais de forma descontrolada e em grandes quantidades para aumentar a captação de usuários que verão as informações. Podem ser considerados maliciosos quando as informações que são bombardeadas na rede são de cunho enganador e difamador, conhecida por fake news.

\section{MATERIAIS E MÉTODOS}

A base de dados escolhida para análise faz referência aos bots sociais da plataforma do Twitter. O objetivo e contribuição deste trabalho é verificar a eficiência dos métodos estatísticos conhecidos como métodos básicos, tais como média, desvio padrão, variância e regressão linear simples, com intuito de poder detectar bots maliciosos do Twitter do tipo falsos seguidores, na tentativa de tornar esse processo mais ágil e automatizado.

\subsection{Ambiente de Desenvolvimento}

A linguagem $\mathrm{R}$ foi escolhida devido a sua facilidade em analisar grandes quantidades de dados, além de conseguir mostrar de forma visual os resultados obtidos. De acordo com Racine (2012) e Team (2000), o R é uma linguagem e um ambiente de desenvolvimento integrado para cálculos e dados estatísticos. Destacam-se como fatores positivos da linguagem $\mathrm{R}$ a variedade técnica de recursos, testes estatísticos clássicos, modelagem de dados lineares e não-lineares, análise de séries temporais e classificação. 


\subsection{Base de Dados}

A base com dados da API (Application Programming Interface) do Twitter é a utilizada para este trabalho. O próprio Twitter fornece acesso aos dados via Standard Search API. A API é responsável pela padronização de transferência dos dados, no caso o Twitter utiliza no formato de texto JSON (JavaScript Object Notation).

A base de dados possui dados capturados de usuários considerados genuínos e de bots sociais, conforme pode ser observado por MIB (2018). A base é composta por arquivos no formato CSV (Comma-separated values), sendo o foco da análise os arquivos de usuários reais e de usuários bots caracterizados como falsos seguidores. A Tabela 1 e 2 apresentam as características dos arquivos.

Tabela 1. Informações quantitativas - usuários reais

\begin{tabular}{cc}
\hline ID & Quantidade \\
\hline Número de registros & 3474 \\
Número de características & 42 \\
\hline
\end{tabular}

Tabela 2. Informações quantitativas - usuários falsos

\begin{tabular}{cc}
\hline ID & Quantidade \\
\hline Número de registros & 3351 \\
Número de características & 38 \\
\hline
\end{tabular}

Para auxiliar os experimentos e igualar as bases, considerou-se apenas características em comum em ambas e que seriam de algum uso para os cálculos estatísticos, assim algumas características que não seriam relevantes para o estudo foram descartadas. A Tabela 3 apresenta as características relevantes para os experimentos.

Tabela 3. Campos relevantes levados em consideração

\begin{tabular}{cc}
\hline Nome & Descrição \\
\hline id & chave de identificação \\
name & nome do usuário \\
screen_name & nome da conta do usuário \\
statuses_count & total de tweets \\
followers_count & quantidade de seguidores \\
friends_count & quantidade de amigos \\
favourites_count & quantidade de favoritos \\
listed_count & quantidade de listas públicas de um usuário \\
created_at & data de criação da conta \\
url & link de referência vinculado à conta \\
lang & linguagem cadastrada \\
time_zone & fuso horário do usuário \\
location & localização do usuário \\
\hline
\end{tabular}

\subsection{Funções Estatísticas}

Recursos pré-compilados e funções estatísticas já desenvolvidas facilitam o estudo da base de dados, centrando a preocupação principal em analisar e verificar o comportamento dos dados.

A fim de tornar a análise menos complexa, principalmente em sua modelagem, optou-se por medidas estatísticas rotuladas como simples, porém justificáveis, já que bots de cunho social se manifestam principalmente por variações dessas medidas.

As funções estatísticas utilizadas se encontram separadas desde cálculos de média e desvio padrão, até testes de hipóteses e regressão linear.

A média tem como objetivo distinguir dados médios de usuários reais e comparar com os dados obtidos em relação aos tipos de bots, no caso de falsos seguidores, dessa forma é possível seguir a análise com outras medidas, consideradas para refinar a comparação. O desvio padrão indica o erro em relação à média de um 
conjunto de dados, o quanto sua característica se alterou em relação ao seu estado padrão, sendo, portanto, uma medida de dispersão. Assim é possível perceber quanto os bots são parecidos ou similares em seus desvios, quanto menor o desvio, maior a estabilidade e menor o erro em relação à média. Por fim, tem-se a variância como outra medida de dispersão, no caso é a raiz quadrada do desvio padrão, indicando a distância do valor para com a média e, quanto menor a variância, menor os valores estarão distantes da média e mais centrado estão os dados.

A Regressão Linear Simples (RLS) estuda a relação entre uma variável chamada de variável dependente e outra chamada de variável independente, sendo que essas duas variáveis no objeto de estudo são variáveis quantitativas. O grau de correlação é medido a partir do coeficiente de regressão linear (cor):

$$
\begin{aligned}
& 0<\operatorname{cor}<0.7: \\
& \operatorname{cor} \geq 0.7: \\
& \operatorname{cor}=0: \\
& -0.7<\operatorname{cor}<0: \\
& \text { cor } \leq-0.7:
\end{aligned}
$$

regressão positiva fraca entre $\mathrm{X}$ e $\mathrm{Y}$ regressão positiva forte entre $\mathrm{X}$ e $\mathrm{Y}$ inexistência da relação linear regressão negativa fraca entre $\mathrm{X}$ e $\mathrm{Y}$ regressão negativa forte entre $\mathrm{X}$ e $\mathrm{Y}$

\subsection{Processamento dos Dados}

Após preparar o ambiente, optou-se por carregar dois arquivos CSV de usuários do Twitter, sendo um com usuários reais (chamado de users) e outro de usuários falsos (chamado de usersfake). A partir desses dois carregamentos, utilizou-se duas funções para extrair informações básicas dos mesmos, tais como, cálculos de médias e desvios.

Para iniciar a análise regressiva, foi necessário alterar informações da base, principalmente se tratando de níveis gráficos e, a fim de encontrar alguma regressão coerente, fez-se diversos testes com as características contáveis das bases.

Uma das manipulações utilizadas foi a de retirar dados nulos ou vazios da base, que muitas vezes não permitia a plotagem de alguns gráficos, principalmente quando as características eram passadas para valores logarítmicos.

Após vários testes com várias características, na busca de algum de possíveis linearidades entre elas, as duas características de quantidade de amigos (friends_count) e quantidade de seguidores (followers_count) se mostraram linearmente dependentes, tendo a característica friends_count como variável resposta e a followers_count como preditora, ou seja, a quantidade de amigos está relacionada linearmente com a quantidade de seguidores que um usuário possui, o que faz sentido lógico, ao aumentar a quantidade de amigos, é natural que haja um aumento da quantidade de seguidores, sendo que por via de regra natural, os amigos também se tornam seguidores.

A partir da descoberta de suposta linearidade entre as variáveis, realizou-se cálculos que puderam mostrar o grau de correlação entre essas variáveis e como elas se comportam em relação a base de usuários falsos (bots). Para tal, usou-se comparações e teste de hipóteses para os cálculos simples e testes de correlação linear para as características que apresentaram suposta linearidade. Para os testes tomou-se:

$$
\begin{array}{ll}
\mathrm{H}_{0}: & \text { Bases iguais }\left(\mathrm{H}_{0}=0\right) \\
\mathrm{H}_{1}: & \text { Bases diferentes }\left(\mathrm{H}_{0} \neq \mathrm{H}_{1}\right)
\end{array}
$$

Para a estatística, a hipótese estabelecida é denominada hipótese nula ou $H_{0}$. Em geral a hipótese é testada como uma hipótese alternativa que é totalmente diferente do resultado dela, ou seja, uma hipótese $H_{l}$. A teoria do teste de hipótese simplesmente busca por regras e procedimentos para dizer se a hipótese nula $\left(H_{0}\right)$ deve ser rejeitada ou não.

Existem duas abordagens para chegar à essa conclusão, seja pelo intervalo de confiança ou pelo teste de significância. Ambas são postuladas da mesma maneira: a variável ou característica em questão tem alguma distribuição de probabilidade e, o teste envolve a formulação de declarações ou afirmações sobre essa variável/característica e seus parâmetros.

Para o teste em questão foi utilizado o método Bicaudal ou Bilateral com o uso do intervalo de confiança de $95 \%$ na qual as hipóteses são extremas (ou igual ou diferente). Foi definido dessa maneira pois a partir dos 
cálculos de médias, desvios e regressão, notou-se diferença expressiva nos resultados, conforme orienta Gujarati e Porter (2011).

Para que as hipóteses serem consideradas diferentes entre si, deve-se analisar o $p$-value, que nada mais é que o valor de probabilidade ou significância assintótica. Esse valor é muito utilizado no contexto de hipótese nula. Se o valor for muito baixo, isto é, se a probabilidade for menor que 5\%, significa que é possível descartar a hipótese nula, portanto há diferença relevante estatisticamente entre as características em análise.

A partir do entendimento dos testes de hipótese, utilizou-se dois testes estatísticos para avaliar o $p$-value necessário e dizer se as características divergem ou não entre as bases. Os códigos do tipo T-Student e de correlação no R foram usados para avaliar o $p$-value necessário e dizer se as características divergem ou não entre as bases. Tais testes devolvem resultados com seu respectivo p-value, porém o teste T-Student normalmente é utilizado em testes de hipóteses simples que não envolvem relações de correlação entre as características, por esse motivo é aplicado o teste de correlação também, o qual analisa amostras emparelhadas. Portanto, o teste T-Student foi escolhido para verificar as hipóteses baseado nas médias, desvios e variâncias das bases. Já o teste de correlação foi utilizado para medir as características que possuem algum grau de correlação, que são citadas anteriormente (friends_count, followers_count, entre algumas outras para comparação).

Segundo Mukaka (2012), para descobrir qual o grau de correlação entre as características, utilizou-se da função que retorna o valor entre os graus citados na Equação I.

\section{RESULTADOS E DISCUSSÃO}

Para os resultados, a priori é mais efetivo analisar os dados mais simples e que podem dar sinais ou indicações mais bruscas de diferenças entre os usuários. Com isso foram calculados as médias, desvios e variâncias das bases e com esse cálculo, realizou-se a comparação utilizando o teste $T$-Student do R, a fim de comprovar se realmente a diferença é estatisticamente relevante. Na sequência são apresentadas as Tabelas comparativas 4,5 e 6 :

Tabela 4. Comparação de médias entre usuários reais e bots do tipo "fake followers"

\begin{tabular}{cccc}
\hline Característica & Reais & Bots & p-value \\
\hline followers_count & 1393.21 & 17.94 & $<2.58^{*} 10^{-6}$ \\
friends_count & 633.24 & 370.05 & $<2.2^{*} 10^{-16}$ \\
statuses_count & 16958.22 & 71.89 & $<2.2^{*} 10^{-16}$ \\
favourites_count & 4669.62 & 4.29 & $<2.2^{*} 10^{-16}$ \\
\hline
\end{tabular}

A Tabela 4 mostra a diferença entre as médias dos usuários reais dos usuários bots. Todas as características quantitativas levadas em consideração foram extremamente relevantes no caso desse tipo de bot ou falsos seguidores. Todos os $p_{-}$values se mostraram reduzidos, menores do que o valor de 0.05 imposto no cálculo padrão da função para aceitar a hipótese $H_{0}$ os quais as características são iguais. Portanto, a partir desse resultado, em relação ao teste de hipótese na qual $H_{1}$ diz que as características são diferentes estatisticamente e como todos os resultados são relevantes, pode-se afirmar que a base de usuários é totalmente diferente uma da outra.

Nesse caso o cálculo de médias e desvios, visualizado na Tabela 5, conseguiram ser eficientes, pois demonstraram uma notável diferença de características quantitativas dos usuários, sendo possível, identificar inúmeras contas que são usadas para dar volume entre outras contas reais no quesito de seguidores e amigos. Esse tipo de conta não se mostra com um padrão humano de utilização do serviço, o que torna fácil a caracterização e identificação desse tipo de usuários.

Tabela 5. Comparação de desvios entre usuários reais e bots do tipo "fake followers"

\begin{tabular}{ccc}
\hline Característica & Reais & Bots \\
\hline followers_count & 17216.66 & 54.22 \\
friends_count & 1600.96 & 212.55 \\
statuses_count & 30696.29 & 634.97 \\
favourites_count & 11527.57 & 59.55 \\
\hline
\end{tabular}


Ao analisar os desvios da Tabela 5, deve-se levar em consideração que como as médias e características são diferentes, os desvios ficaram bem diferentes entre si.

Mais uma vez é possível ver como os usuários reais divergem dos bots, com grandes diferenças, o que significa que os dados estão mais dispersos, isto mostra novamente o comportamento imprevisível humano como fator dos altos desvios em relação à pouca mudança ou alteração dos bots, também observado pela Tabela 6, que mostra a comparação das variâncias.

Tabela 6. Comparação de variâncias entre usuários reais e bots do tipo "fake followers"

\begin{tabular}{ccc}
\hline Característica & Reais & Bots \\
\hline followers_count & 296413537 & 2939.372 \\
friends_count & 2563082 & 45177.68 \\
statuses_count & 942261981 & 403199.2 \\
favourites_count & 132884793 & 3546.381 \\
\hline
\end{tabular}

\subsection{Regressão Linear Simples - Usuários Reais}

A fim de encontrar características lineares entre si e compará-las com a mesma segmentação dos bots, optou-se por buscar e plotar gráficos relacionados, juntamente com seu grau de correlação, podendo assim dizer se as várias características escolhidas conseguem atingir um grau de correlação satisfatório para a pesquisa.

Algumas mudanças na análise dos dados também foram necessárias, principalmente para encontrar alguma correlação entre as variáveis. Essas mudanças estão relacionadas em alterar as características para a função logarítmica, muito utilizada estatisticamente para grande volume de dados. A partir da Figura 2, é possível verificar as características mais relevantes para a pesquisa:

Para uma melhor visualização preferiu-se o uso da função logarítmica em ambas as características, como mostra a Figura 1, podemos observar um gráfico mais coeso e passível de análise. Cabe ressaltar que mesmo alterando ambas as características para logaritmo, não há problemas nos cálculos realizados, pois não há alteração da análise.

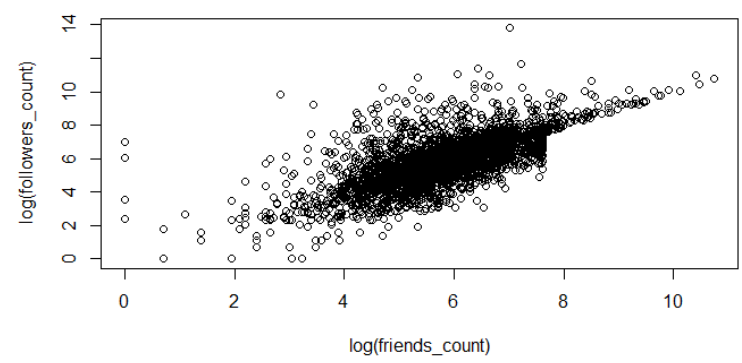

Figura 1. Plot(log(followers_count), $\log ($ friends_count $))$ - Usuários reais

Retornando a primeira análise, é possível perceber uma certa correlação entre as características followers_count e friends_count, mas para isso é necessário também calcular qual é a melhor reta que passa pelos pontos plotados e a função que traça o modelo linear baseado nas características plotadas. A partir da Figura 2, é possível iniciar o teste de correlação para verificar em qual grau as características se encontram: 


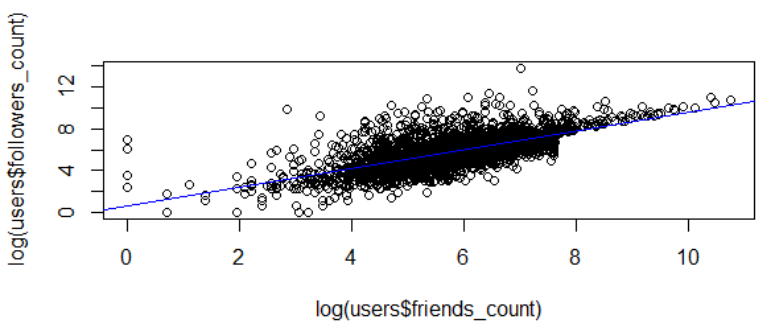

Figura 2. Aplicação da função "lm()" e "abline()" - Regressão Linear

A partir da Figura 2, é possível descrever a Equação da reta que passa pelos pontos, dada por:

$$
f(x)=0.6660+0.8938 x
$$

Na sequência, é necessário analisar a correlação entre as características, o qual resultou em uma regressão com grau de correlação de $68.85 \%$, sendo, portanto, uma regressão positiva fraca, menor que 0.7 , porém muito próximo de ser forte, o que se torna uma análise válida, ainda mais por estar usando dados brutos.

A seguir analisou-se graficamente todas as características relevantes para este trabalho, followers_count e statuses_count (quantidade de tweets já postados), followers_count e favourities_count (quantidade de favoritos do usuário), todas sendo manipuladas da mesma forma que a primeira, ou seja, utilizando-se da mudança para logaritmo.

Da mesma forma como analisado anteriormente, foi possível construir uma análise também dos dados relacionados ao número de seguidores com a quantidade de tweets postados por usuário, conforme visto por meio da Figura 3.

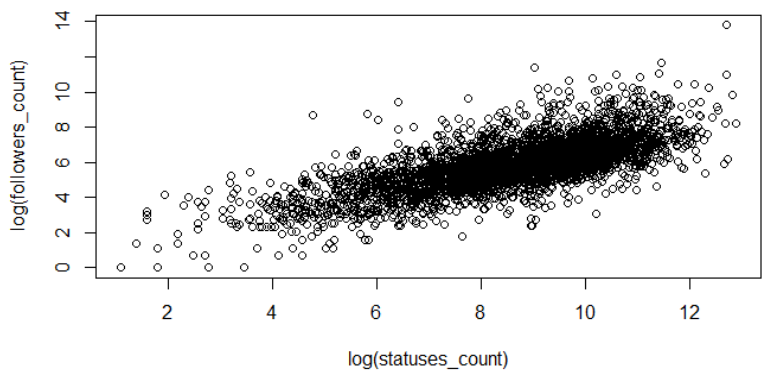

Figura 3. Quantidade de seguidores x Quantidade de Tweets - Usuários reais

Função do modelo linear adotado pelo gráfico da Figura 4:

$$
f(x)=0.9969+0.5655 x
$$

A partir da distribuição e da plotagem da Figura 4, é preciso analisar a correlação entre os dados. Nesse caso o valor de correlação entre os dados é de $71.15 \%$, sendo que, por estar acima de 0.7 é considerada uma correlação positiva forte. Dessa forma, também é uma correlação válida. 


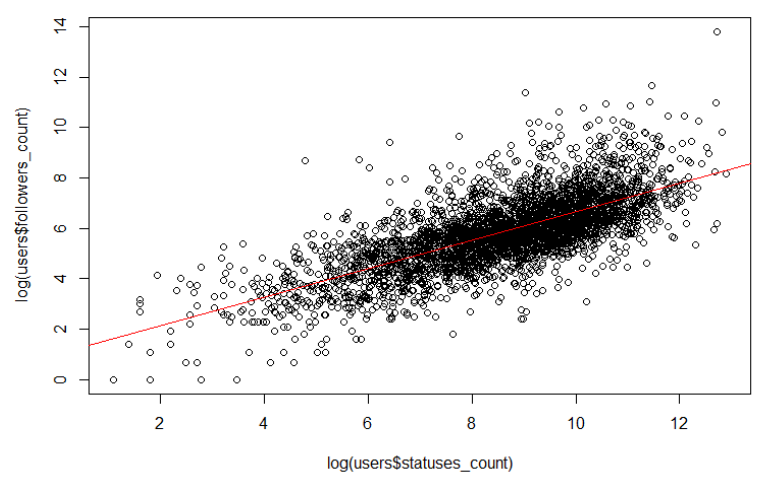

Figura 4. Modelo Linear Seguidores x Tweets - Usuários reais

Outra análise para a base de dados reais, está relacionada com o grau de correlação da quantidade de seguidores e a quantidade de tweets favoritados pelo usuário, conforme apresentado pela Figura 5:

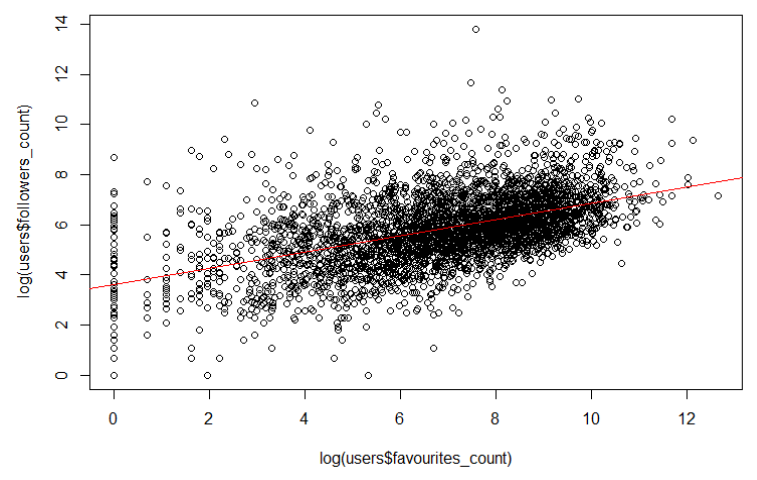

Figura 5. Modelo Linear Seguidores x Favoritos - Usuários reais

O grau de correlação nesse sentido foi de apenas 0.4956 , o que leva a dizer que existe uma correlação fraca entre essas duas características, não sendo necessariamente relevante para este estudo, porém foi utilizada em níveis comparativos com a base de usuários bots do tipo de seguidores falsos.

\subsection{Regressão Linear Simples - Usuários Bots}

O mesmo procedimento realizado para os usuários reais e em busca de encontrar diferenças entre a base de bots e a real, realizou-se para a base de usuários do tipo de seguidores falsos. Seguindo as mesmas características e os mesmos procedimentos, obteve-se um resultado para os níveis de correlação entre os dados. O objeto central de estudos encontra-se nessa regressão, portanto ela é essencial e possui um bom grau de relevância para detectar anomalias no padrão dos bots do tipo fake followers. Analogamente ao que foi realizado com a base de usuários reais, fez-se com a base de bots do tipo citado anteriormente, em busca de semelhanças e principalmente de diferenças visíveis e/ou calculáveis. Seguindo com o objeto de estudo tem-se a imagem da relação das características mostradas nas Figuras 6 e 7: 


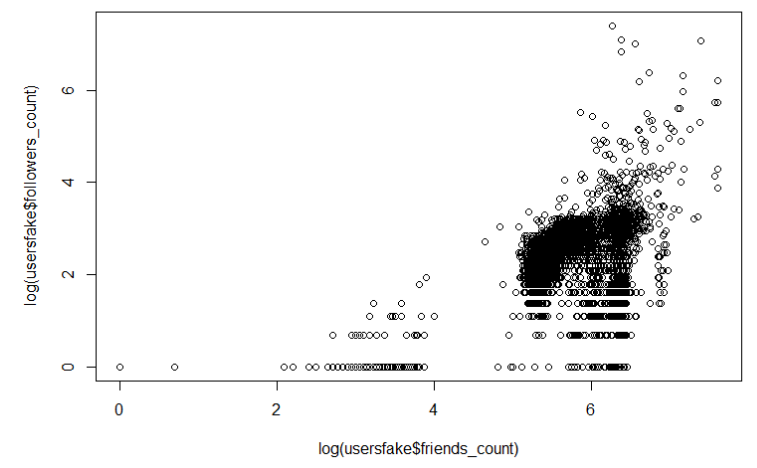

Figura 6. Quantidade de seguidores x Quantidade de amigos - Usuários bots

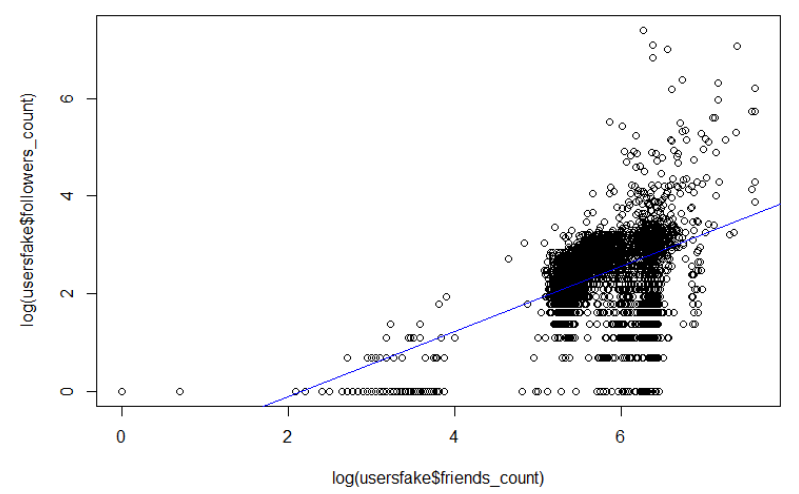

Figura 7. Modelo Linear Seguidores x Amigos - Usuários bots

Observando a Figura 6 em relação a Figura 2 para usuários reais, há uma aglomeração de pontos em algumas regiões, e nenhum ponto em outras. É notado, portanto, uma correlação entre as características, pois não é possível visualizar nitidamente uma reta atravessando esses pontos como ocorre na Figura 3, que apesar de possuir um corpo mais curvado, também possui um alongamento maior em relação a Figura 6. Na sequência calculou-se a Equação da reta para essas duas características. Tem-se sua fórmula matemática dada pela Equação V:

$$
f(x)=-1.4464+0.6686 x
$$

Em relação ao seu grau de correlação, que aparentemente parece ser baixo, pode-se dizer que não se aproximou da correlação para usuários reais com um total de 0.5435 ou seja, é uma regressão linear fraca com um grau de correlação entre as características de $54.35 \%$. Percebe-se uma diferença entre os usuários reais, tal que será discutida mais à frente. Comparativamente, a análise representada na Figura 9 mostra a diferença entre as características de quantidade de seguidores e quantidade de tweets, buscando uma correlação entre elas. Comparando com a base de usuários reais a fim de perceber grandes mudanças com essa regressão, é possível dizer que à medida que os seguidores aumentam, a quantidade de postagem necessariamente aumenta também, não sendo, claro uma via de regra, porém uma possibilidade. 


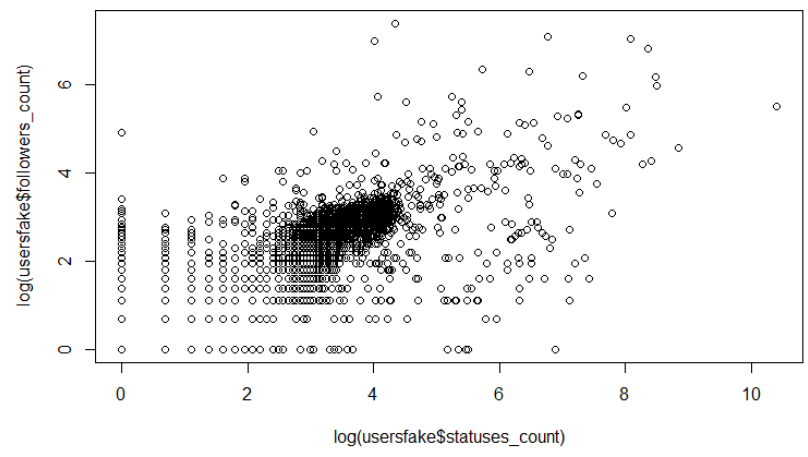

Figura 8. Quantidade de seguidores x Quantidade de Tweets - Usuários bots

Vemos também uma diferença em relação ao mesmo gráfico de usuários reais, na Figura 8 os pontos estão distribuídos uniformemente, porém com concentração de pontos em certa localização, comparado com o de usuários reais, não há tantas aglomerações e está bem mais uniformemente distribuído. A possibilidade para este resultado é de que os usuários bots, apesar de seguirem padrões humanos dentro do Twitter, não conseguem manter uma linearidade na quantidade de tweets postados com a quantidade de seguidores que possuem, visto que sua média também segue um padrão muito menor nas postagens. $\mathrm{O}$ modelo linear é montado e descrito como mostrado na Figura 9.

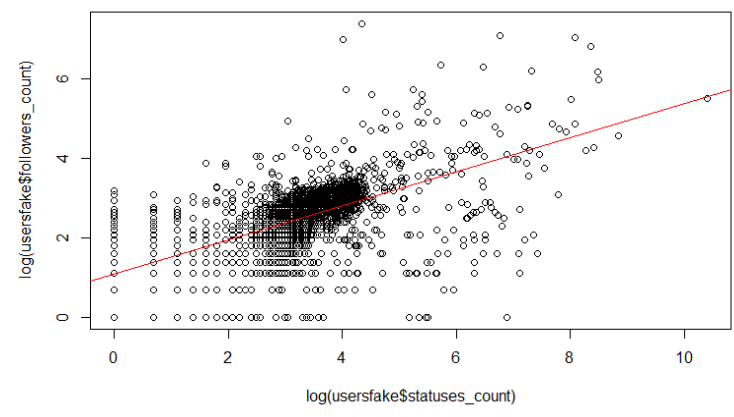

Figura 9. Modelo Linear Seguidores x Tweets - Usuários bots

Seguindo com os cálculos, tem-se que a Equação da reta desse modelo é dada por:

$$
f(x)=1.0751+0.4312 x
$$

Dadas as informações obtidas pelas características e calculando o grau de correlação tem-se como resultado o valor 0.6360 que representa uma correlação fraca entre os dados. Comparada com o cálculo da correlação de usuários reais, que é de uma correlação forte, percebe-se uma diferença grande em relação aos cálculos.

Como última análise de regressão linear, porém não menos importante, tem-se as características de seguidores por quantidade de tweets marcados como favoritos. Essa relação tenta mostrar que a quantidade de seguidores ao aumentar, aumenta a quantidade de tweets marcados como favoritos pelos seus seguidores, o que de fato é lógico, visto que a tendência de tweets marcados como favoritos tende a crescer com o aumento de seguidores. Porém, analisando os resultados da Figura 10 tem-se um fator não linear e pouco coeso: 


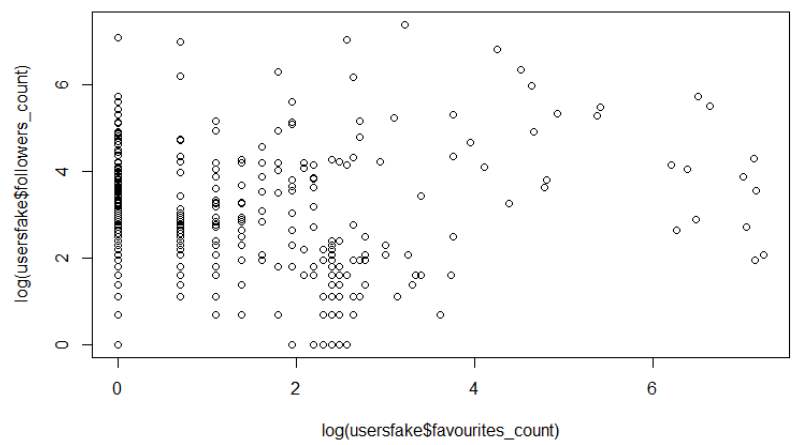

Figura 10. Quant. de Seguidores x Quant.de Favoritados - Usuários bots

É possível perceber que a correlação é praticamente inexistente, não segue um padrão ou linearidade, mas para fins de comprovação, calculou-se o modelo linear, mostrado na Figura 11:

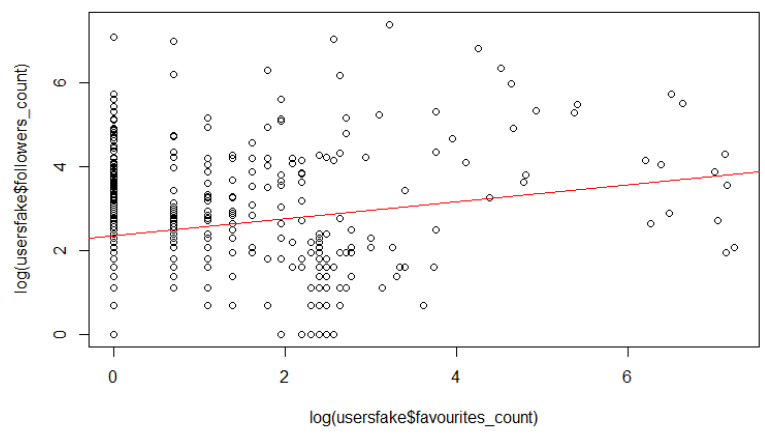

Figura 11. Modelo Linear Seg. x Tweets marcados como fav. - Usuários bots

A partir do modelo linear apresentado na Figura 13, a Equação da reta plotada é dada por:

$$
f(x)=2.3484+0.2033 x
$$

A partir das informações obtidas pelas Figuras 11 e 12 e calculando a correlação das duas características, tem-se como resultado um grau de correlação de 0.1514 , ou seja, extremamente fraco e positivo, o que leva o não descarte da mesma correlação de usuários reais, que apesar de ser fraca, é três vezes maior que a mesma de usuários bots. Portanto, é possível afirmar que obter dados expressivos na deteçãa de bots por meio da estatística é algo realizável, sendo que isso é apenas uma pequena parte de todas as análises e comparações possíveis.

A fim de mostrar a utilidade estatística na detecção, as características citadas foram escolhidas como principal fonte de estudo, porém outras mais poderiam ser escolhidas, como por exemplo uma análise de quantidade de amigos com todas as outras que foram utilizadas junto com a quantidade de seguidores. As possibilidades de estudo são amplas.

\section{CONCLUSÃO}

Após os dados analisados é possível apresentar informações relevantes da utilização da estatística para o cálculo e detecção de bots, do tipo Social Bots, mais especificamente os bots considerados por falsos seguidores, responsáveis apenas para indicar a falsa impressão de que alguns usuários possuem grande popularidade na rede. 
Por meio da estatística básica, com o cálculo de médias e desvios, se mostrou muito eficiente quando levado em comparação os usuários reais de bots. Com o teste de hipótese, podemos complementar mais a pesquisa, oferecendo maior veracidade nas características analisadas, mostrando que são totalmente diferentes entre si, já que seu p-value é extremamente pequeno, mais uma vez fortalecendo o fato da hipótese nula $H_{0}$ ser falsa, aceitando-se $H_{l}$ além de todas as características quantitativas serem diferentes, referenciando que as bases não são iguais e possui diferenças em suas medidas.

Por fim, a regressão linear, método para mostrar o grau de afinidade e interferência entre as características, que demonstraram uma maior linearidade quando testadas com usuários reais, além de certos padrões nos pontos dos gráficos, não podendo ser dito o mesmo para regressões dos usuários bots, que apresentaram uma maior deformidade nos gráficos, consequentemente uma correlação mais fraca.

Vale a pena ressaltar que a regressão realizada serviu para demonstrar o quanto pode ser útil, provavelmente existem muito mais correlações entre as variáveis, levando até um estudo mais aprofundado sobre o contexto para trabalhos futuros, o que pode mostrar mais resultados comparativos entre bots dessa categoria e usuários reais.

\section{REFERÊNCIAS}

Broniatowski, D. et al., 2018. Weaponized health communication: Twitter bots and Russian trolls amplify the vaccine debate. American journal of public health, 108(10), 1378-1384.

Campos, G. F. et al., 2018. Detection of human, legitimate bot, and malicious bot in online social networks based on wavelets. ACM Transactions on Multimedia Computing, Communications, and Applications (TOMM), 14(1s), 26.

Carna Botnet., 2012. Avaliable in: <http://census2012.sourceforge.net/paper.html> Access Date: 24/03/2018. 2018.

Chavoshi, N. et al., 2016a. DeBot: Twitter Bot Detection via Warped Correlation. In ICDM (pp. 817-822).

Chavoshi, N. et al., 2016b. Identifying correlated bots in twitter. In International Conference on Social Informatics (pp. 14-21). Springer, Cham.

Chen, Z. et al., 2017. Hunting Malicious Bots on Twitter: An Unsupervised Approach. In International Conference on Social Informatics (pp. 501-510). Springer, Cham.

Davis, C. et al., 2016. Botornot: A system to evaluate social bots. In Proceedings of the 25th International Conference Companion on World Wide Web (pp. 273-274). International World Wide Web Conferences Steering Committee.

Distil's Bad Bot Report 2018, 2018. Avaliable in: <https://resources.distilnetworks.com/all-blog-posts/bad-bot-reportnow-available> Access Date: 28/07/2018. 2018.

Efthimion, P. et al., 2018. Supervised machine learning bot detection techniques to identify social twitter bots. In SMU Data Science Review, 1(2), 5.

Gujarati, D. N., Porter, D. C., 2011. Econometria Básica-5. Amgh Editora.

Haidermota, M., Pansare, A., 2018. Classifying twitter user as a bot or not and comparing different classification algorithms. In International Journal of Advanced Research in Computer Science, 9(3), 29.

Howard, P. et al., 2016. Bots and automation over Twitter during the third US presidential debate.

MIB: enhancing the user awareness on Search Engines and Social Media., 2018. Avaliable in: <http://mib.projects.iit.cnr.it/index.html> Access Date: 20/09/2018. 2018.

Morstatter, F. et al., 2016. A new approach to bot detection: striking the balance between precision and recall. In 2016 IEEE/ACM International Conference on Advances in Social Networks Analysis and Mining (ASONAM) (pp. 533-540). IEEE.

Mukaka, M. M., 2012. A guide to appropriate use of correlation coefficient in medical research. In Malawi Medical Journal, 24(3), 69-71.

Qamar, A., Karim, A., \& Chang, V. (2019). Mobile malware attacks: Review, taxonomy \& future directions. Future Generation Computer Systems, 97, 887-909.

Racine, J. S., 2012. RStudio: a platform-independent IDE for R and Sweave. In Journal of Applied Econometrics, 27(1), 167-172.

Team, R. C., 2000. R language definition. Vienna, Austria: R foundation for statistical computing. 